Z Gerontol Geriat 2016 · 49:350

DOI 10.1007/s00391-016-1076-3

Online publiziert: 3. Juni 2016

๑) Springer-Verlag Berlin Heidelberg 2016

CrossMark

\section{Originalpublikation}

Satizabal CL, Beiser AS, Chouraki V, Chêne G, Dufouil C, Seshadri S (2016) Incidence of dementia over three decades in the Framingham Heart Study. N Engl J Med 374:523-532

Einleitung. Die Abhängigkeit von Pflegepersonen und körperliche Behinderungen werden häufig durch eine Demenzerkrankung verursacht. Da bisherige Untersuchungen zur Inzidenz der Erkrankung widersprüchlich waren, wurden die Teilnehmer der Framingham Heart Study-einer epidemiologischen Studie über 3 Dekaden - untersucht.

Methode. Die ursprüngliche Studie war 1948 initiiert worden. Im Ort Framingham, Massachusetts, wurden alle Bewohner alle 2 Jahre auf Gesundheitsparameter untersucht. Seit 1971 wurden auch die Nachkommen einbezogen. Im Detail wurden in dieser Kohorte neben allgemeinen Untersuchungen $\mathrm{zu}$ Gesundheitsindikatoren neuropsychologische Tests (Mini-Mental State Examination, MMSE) durchgeführt. Im Verdachtsfall einer Demenz stellten Psychiater die Diagnose gemäß den Kriterien des Diagnostic and Statistical Manual of Mental Disorders.

Resultate. Insgesamt wurden 40.192 Personenjahre im Beobachtungszeitraum von 1971 bis 2007 in die Analyse einbezogen. Das Durchschnittsalter der Studienteilnehmer betrug 72 Jahre in den letzten 3 Epochen der Untersuchung; es waren $57 \%$ Frauen. Die MMSE-Werte waren im Beobachtungszeitraum gleich. Die allgemeinen Gesundheitsindikatoren waren im Verlauf vergleichbar; der Bildungsstatus stieg leicht. Die Häufigkeit der speziellen kardiovaskulären

\author{
P. Dovjak \\ Salzkammergut-Klinikum, Gmunden, Österreich
}

\title{
Sinkende Inzidenz der Demenz
}

Risikofaktoren wie Übergewicht und Diabetes stieg, die der übrigen Risikofaktoren dafür sank. Insgesamt wurden 371 Demenzerkrankungen diagnostiziert; das Alter der Studienteilnehmer bei Diagnosestellung stieg im Beobachtungszeitraum von 80 Jahren zu Beginn auf 85 Jahre in der 4. Periode der Untersuchung signifikant an. Die Risikorate für die Demenzerkrankung sank von einer „hazard ratio“ (HR) 3,6 (Konfidenzintervall [KI] 2,9-4,4) pro 100 Personen in der ersten Periode der Untersuchung auf HR 2,0 (KI 1,5-2,6) in der letzten Untersuchungsperiode und damit um $20 \%$ durchschnittlich pro Dekade. Dieser Rückgang wurde bei der vaskulären Demenz und nichtsignifikant bei der Demenz vom Alzheimer-Typ gefunden.

Diskussion. Die Daten zeigen einen progressiven Rückgang der Inzidenz an Demenzerkrankungen bei den Teilnehmern der Framingham Heart Study. Besonders bei Absolventen höherer Bildung war dieser Trend zu beobachten. Die Studienautoren deuteten die verbesserte Behandlung von kardiovaskulären Risiken als Ursache dieses Trends und auch die geringere Inzidenz an neurodegenerativen Veränderungen durch den Rückgang der kardiovaskulären Risiken.

\section{Kommentar}

Diese beeindruckenden Studienergebnisse stehen im Konsens mit vorhergehenden Arbeiten zum Thema in Großbritannien und den Niederlanden [1, 2, 3]. Es sind Ergebnisse einer langen Beobachtungszeit in einer großen Studienkohorte. Einschränkend ist die Fokussierung auf Menschen europäischer Abstammung $\mathrm{zu}$ werten. Der positive Trend ist natürlich im Zusammenhang mit der entgegenwirkenden demografi- schen Entwicklung der älter werdenden Bevölkerung in den westlichen Ländern $\mathrm{zu}$ betrachten.

\section{Fazit für die Praxis}

Obwohl die Prävalenz der Demenzerkrankten aufgrund der demografischen Entwicklung steigt, sinkt die Inzidenz durch die medizinische Behandlung. Die medizinischen Interventionen kardiovaskulärer Risiken zeigen Wirkung und bleiben für die Prävention von Demenzerkrankungen wichtig.

\section{Korrespondenzadresse}

Prim. Dr. P. Dovjak

Salzkammergut-Klinikum

Miller-von-Aichholz-Str. 49, 4810 Gmunden Österreich

peter.dovjak@gespag.at

Interessenkonflikt. P. Dovjak gibt an, dass kein Interessenkonflikt besteht.

\section{Literatur}

1. Qiu C, Strauss E von, Bäckman L, Winblad B, Fratiglioni L (2013) Twenty-year changes in dementia occurrencesuggest decreasing incidence in centra Stockholm, Sweden. Neurology 80:1888-1894

2. Matthews F, Athur E, Barnes L, Bond J, Jagger C, Robinson L, Brayne C (2013) A two-decade comparison of prevalence of dementia in individuals aged 65 years and older from three geographical areas of England: results of the cognitve function and ageing study I and II. Lancet 382: 1405-124.

3. Schrijvers E, Verhaaren B, Koudstaal P, Hofman A, Ikram M, Breteler M (2012) Is dementia incidence declining?: Trends in dementia incidence since 1990 in the Rotterdam study 